\title{
Propagation of Electrostatic Solitary Wave Structures in Dense Astrophysical Plasma: Effects of Relativistic Drifts \& Relativistic Degeneracy Pressure
}

\author{
Swarniv Chandra \\ ${ }^{1}$ Department of Physics, JIS University, Kolkata-700109, India \\ ${ }^{2}$ Department of Physics, Jadavpur University, Kolkata-700032, India \\ Email: swarniv147@gmail.com
}

\begin{abstract}
Analytical and numerical studies are presented for electron acoustic solitary wave structure in relativistic degenerate two-component unmagnetized astrophysical plasma. The existence of a wave mode of pure quantum origin is predicted. The effect of various plasma parameters on the conditions of existence and properties of solitary wave is investigated. It is shown that depending on the values of plasma parameters both rarefactive and compressive type of solitons can exist. It is observed that the amplitude and width of the solitons are significantly affected by the quantum and relativistic effects. The relativistic effects arising out of streaming motion is treated by Eulerean formulation whereas the relativistic degeneracy effects is investigated by making use of Chandrasekhar formula.
\end{abstract}

Keywords: Quantum plasmas, astrophysical plasma, relativistic degeneracy, quantum diffraction, solitary structures, quantum hydrodynamic model.

\section{Introduction}

In recent years there has been a great deal of interest in studying the different aspects of nonlinear wave propagation in quantum plasma. Traditional plasma physics has mainly focused on regimes characterized by high temperature and low density where quantum effects have virtually no impact. But in plasmas where the density is quite high and the temperature is very low thermal de Broglie wavelength of electrons may become comparable to the spatial scales of the system and then quantum nature of the plasma constituents cannot be neglected and quantum effects could affect the plasma behavior in a significant way. The condition is well satisfied in some compact astrophysical objects (e.g. white dwarfs, neutron stars, magnetars etc.), also in metals, semiconductors and laser produced plasmas so that such phenomenon may be observed. The matter exists in extreme conditions of density. In such situation the average inter-Fermion distance is comparable to or less than the thermal de Broglie wavelength and hence quantum degeneracy effects become important. Quantum effect arises due to overlapping of the wavefunctions of the neighboring particles. In quantum plasmas where the electron thermal energy is much smaller than their Fermi energy the statistical behavior of plasma particles should be described by Fermi-Dirac statistics and not by the classical Boltzmann statistics. Such quantum plasmas may be found in a variety of environments such as metal nanostructures [1], astrophysical system [2], ultra-small electronic devices [3,4], biophotonics [5], cool vibes [6], neutron stars [7], laser produced plasmas [8], quantum wells and quantum diodes [9,10].

Most of the investigations on nonlinear wave propagation in quantum plasma are confined to the nonrelativistic case. But when the electron or ion velocity approaches the speed of light relativistic effect may significantly modify the nonlinear behavior of electron plasma waves. Relativistic plasma can be formed in many practical situations, e.g. in space-plasma phenomena $[2,7]$, the plasma sheet boundary of earth's magnetosphere [11, 12], van Allen radiation belts [13] and laser-plasma interaction experiments $[14,15]$. The relativistic motion in plasmas is also assumed to exist during the early period of evolution of the universe [16]. Regarding the relativistic effects on ion-acoustic solitary waves a number of works have been reported for classical plasma. For example, Saeed et al [17] have shown that 
in electron-positron-ion plasma increase in the relativistic streaming factor causes the soliton amplitude to thrive and its width shrinks. El-Labany et al [18] have shown that relativistic effect can modify the condition of modulational instability of ion-acoustic waves in a warm plasma with nonthermal electrons. Regarding the relativistic effects on electron plasma waves only a very few works can be found in the literature. Recently Bharuthram and $\mathrm{Yu}[19]$ have shown that relativistic electron plasma waves can propagate as quasi-stationary nonlinear waves as well as solitary waves. So far all the works on the relativistic effects on plasma waves have been reported for classical plasma. In course of the interesting developments of quantum effects in plasma it is natural to investigate whether the combined effects of relativity and quantum mechanics can display some properties of plasma. Relativistically degenerate dense plasmas can be found in many astrophysical environments including interiors of white dwarf stars [20] and magnetars [21]. Thus it is important to investigate the relativistic and quantum mechanical effects in a combined way on the nonlinear wave structure in plasma.

The inclusion of quantum-mechanical effects in plasma requires new mathematical formulation or a suitable modification of the formulations used in classical situations. Quantum effects in plasmas are usually studied with the help of two well-known formulations, viz. the Wigner-Poisson and the Schrödinger-Poisson formulations. The Wigner-Poisson model is often used in the study of quantum kinetic behavior of plasma. The Schrodinger-Poisson model describes the hydrodynamic behavior of plasma particles in quantum scales. It can be considered as the quantum analog of the fluid model of traditional plasma. The quantum hydrodynamic (QHD) model is derived by taking velocity-space moments of the Wigner equations as in the classical fluid model. This model consists of a set of equations describing the transport of charge, momentum and energy in a charged particle system interacting through a self-consistent electrostatic potential. The QHD model generalizes the fluid model for plasma with the inclusion of a quantum correction term also known as the Bohm potential [22]. The model incorporates quantum statistical effects through an equation of state. The quantum corrections may give rise to new aspects of purely quantum origin in the collective behavior of plasma at quantum scale. For example, it may lead to the generation of new wave modes in plasma [23]. Because of simplicity, straight forward approach and numerical efficiency the QHD model has been widely used by several authors [24-31] in dealing with different aspects of linear and nonlinear wave propagation in unmagnetized quantum plasmas. For example, using the QHD model Haas et al [22] have studied the important role of quantum diffraction in linear as well as nonlinear regimes for the propagation of ion acoustic waves; Gardner and Ringhofer [24] has studied the electron-hole dynamics in semiconductors. Using the same model Shukla and Eliasson [25] have studied the dynamics and formation of dark soliton and vortices in quantum plasma. It has also been used to study the Korteweg deVries (KdV) solitary wave structure for ion acoustic waves [26, 27], electron-acoustic waves [28], dust-acoustic waves and dust ion-acoustic waves $[29,30]$. Recently we have studied the effect of quantum diffraction on the electron plasma waves and it has been found that quantum effects can significantly modify the modulational instability conditions and the instability growth rates of finite amplitude electron plasma waves [31].

There are practical situations such as intense laser-solid interaction experiments and presumably the early period of the evolution of the universe where both the quantum and relativistic effects may become important for consideration. The motivation of the present paper is to study the relativistic effect on the formation and characteristics of KdV solitary wave structure of plasma waves in a degenerate plasma including full ion dynamics. Using the one-dimensional quantum hydrodynamic (QHD) model for two component electron-ion dense quantum plasma we have studied the linear and nonlinear properties of a plasma wave mode of pure quantum origin including relativistic effects. Because of heavier mass quantum correction for ions is smaller than that for electrons. However to make a complete analytical study we have considered, unlike previous authors, quantum corrections for both the electrons and ions. It is shown that the relativistic effects can significantly change the linear and nonlinear properties of the waves in quantum plasma.

The paper is organized in the following way: in section 2 the basic set of quantum hydrodynamic equations are presented including streaming motion; in section 2.1 the linear dispersion characteristics and existence of a wave mode of pure quantum origin is predicted; in section 2.2 the Korteweg deVries equation is derived by using the standard perturbation techniques. In section 3 the formulation for relativistic degeneracy is introduced and the basic set of equations are written, section 3.1 deals with the linear dispersion characteristics, in section 3.2 the solitary profiles are investigated. Section 4 gives a comparative study of these two kind of relativistic effects. 


\section{Basic Equations}

We consider relativistic dense quantum plasma consisting of electrons and ions with a streaming motion along the x-axis. We also assume that the plasma particles behave as a one dimensional Fermi gas at zero temperature and therefore the pressure law [22] is:

$$
p_{j}=\frac{m_{j} V_{F j}^{2}}{3 n_{j 0}^{2}} n_{j}^{3}
$$

where $j=e$ for electron and $j=i$ for ions; $m_{j}$ is the mass; $V_{F j}=\sqrt{2 k_{B} T_{F j} / m_{j}}$ is the Fermi thermal speed, $T_{F j}$ is the Fermi temperature and $k_{B}$ is the Boltzmann constant; $n_{j}$ is the number density with the equilibrium value $n_{j 0}$. The set of QHD equations describing the dynamics of the electron plasma waves in the model plasma under consideration are the following:

$$
\begin{gathered}
\frac{\partial n_{j}}{\partial t}+\frac{\partial\left(n_{j} \gamma_{j} u_{j}\right)}{\partial x}=0 \\
\left(\frac{\partial}{\partial t}+u_{j} \frac{\partial}{\partial x}\right)\left(u_{j} \gamma_{j}\right)=-\frac{q_{j}}{m_{j}} \frac{\partial \phi}{\partial x}-\frac{1}{m_{j} n_{j}} \frac{\partial p_{j}}{\partial x}+\frac{\hbar^{2}}{2 m_{j}^{2} \gamma_{j}} \frac{\partial}{\partial x}\left[\frac{1}{\sqrt{n_{j}}} \frac{\partial^{2} \sqrt{n_{j}}}{\partial x^{2}}\right] \\
\frac{\partial^{2} \phi}{\partial x^{2}}=4 \pi \sum_{j} q_{j} \gamma_{j} n_{j}
\end{gathered}
$$

where $u_{j}, q_{j}$ and $p_{j}$ are respectively the fluid velocity, charge and pressure of the $j^{\text {th }}$ species, $q_{e}=-e, q_{i}=e, \gamma_{j}=\left(1-u_{j}^{2} / c^{2}\right)^{1 / 2}$ is the relativistic factor, $c$ is the velocity of light in free space, $\hbar$ is the Planck's constant divided by $2 \pi$ and $\phi$ is the electrostatic wave potential. We now use the following normalization:

$$
x \rightarrow x \omega_{p e} / V_{F e}, t \rightarrow t \omega_{p e}, \phi \rightarrow e \phi / 2 k_{B} T_{F e}, n_{j} \rightarrow n_{j} / n_{0} \text { and } u_{j} \rightarrow u_{j} / V_{F e}
$$

where $\omega_{p e}=\sqrt{4 \pi n_{0} e^{2} / m_{e}}$ is the electron plasma oscillation frequency and $V_{F e}$ is the Fermi thermal speed of electrons. The normalization gives us the following simplified set of equations for electrons and ions as:

$$
\begin{gathered}
\frac{\partial\left(n_{e}\right)}{\partial t}+\frac{\partial\left(n_{e} \gamma_{e} u_{e}\right)}{\partial x}=0 \\
\left(\frac{\partial}{\partial t}+u_{e} \frac{\partial}{\partial x}\right)\left(u_{e} \gamma_{e}\right)=\frac{\partial \phi}{\partial x}-n_{e} \frac{\partial n_{e}}{\partial x}+\frac{H^{2}}{2 \gamma_{e}} \frac{\partial}{\partial x}\left[\frac{1}{\sqrt{n_{e}}} \frac{\partial^{2} \sqrt{n_{e}}}{\partial x^{2}}\right] \\
\frac{\partial n_{i}}{\partial t}+\frac{\partial\left(n_{i} \gamma_{i} u_{i}\right)}{\partial x}=0 \\
\left(\frac{\partial}{\partial t}+u_{i} \frac{\partial}{\partial x}\right)\left(u_{i} \gamma_{i}\right)=-\mu \frac{\partial \phi}{\partial x}-\sigma n_{i} \frac{\partial n_{i}}{\partial x}+\frac{\mu^{2} H^{2}}{2} \frac{\partial}{\partial x}\left[\frac{1}{\sqrt{n_{i}}} \frac{\partial^{2} \sqrt{n_{i}}}{\partial x^{2}}\right] \\
\frac{\partial^{2} \phi}{\partial x^{2}}=\left(\gamma_{e} n_{e}-\gamma_{i} n_{i}\right)
\end{gathered}
$$

where $H=\hbar \omega_{p e} / 2 k_{B} T_{F e}$ is a nondimensional quantum parameter proportional to the quantum diffraction, $\mu=m_{e} / m_{i}$ is the ratio of electron and ion mass and $\sigma=T_{F i} / T_{F e}$ is the ratio of ion and electron Fermi temperatures. The quantum diffraction parameter $H$ is proportional to the ratio between the plasmon energy $\hbar \omega_{p e}$ (energy of an elementary excitation associated with an electron plasma wave) and the Fermi energy $k_{B} T_{F e}$. The equations (5)-(9) constitute the basic set of quantum hydrodynamic equations to be used in the investigation of nonlinear propagation of electron plasma 
waves in quantum plasma. Quantum effects are included in the model through the second and third terms on the R.H.S of equations (6) and (8). The second term on the RHS of Equations (6) and (8) include quantum statistical effect through the equation of state [Eq. (4)]. The third term in the RHS of Equations (6) and (8) arises due to quantum correction of density fluctuations and this type of quantum effect is called quantum diffraction or Bohm potential.

\subsection{Linear Dispersion Characteristics}

In order to investigate the nonlinear behavior of plasma waves we make the following perturbation expansion for the field quantities $n_{e}, u_{e}, n_{i}, u_{i}$ and $\phi$ about their equilibrium values:

$$
\left[\begin{array}{c}
n_{e} \\
u_{e} \\
n_{i} \\
u_{i} \\
\phi
\end{array}\right]=\left[\begin{array}{c}
1 \\
u_{0} \\
1 \\
u_{0} \\
0
\end{array}\right]+\varepsilon\left[\begin{array}{c}
n_{e}^{(1)} \\
u_{e}^{(1)} \\
n_{i}^{(1)} \\
u_{i}^{(1)} \\
\phi^{(1)}
\end{array}\right]+\varepsilon^{2}\left[\begin{array}{c}
n_{e}^{(2)} \\
u_{e}^{(2)} \\
n_{i}^{(2)} \\
u_{i}^{(2)} \\
\phi^{(2)}
\end{array}\right]+\ldots \ldots
$$

Assuming that all the field quantities are varying as $e^{i(k x-\omega t)}$, we get for normalized wave frequency $\omega$ and wave number $k$, the following dispersion relation of electron plasma waves which includes quantum and relativistic effects for both electrons and ions:

$$
\frac{1}{\left(\omega-k u_{0}\right)^{2} \gamma_{3}-k^{2}-\frac{H^{2} k^{4} \gamma_{0}}{4}}+\frac{\mu}{\left(\omega-k u_{0}\right)^{2} \gamma_{3}-\sigma k^{2}-\frac{\mu^{2} H^{2} k^{4} \gamma_{0}}{4}}=1
$$

where $\gamma_{3}=1+\frac{3 u_{0}^{2}}{2 c^{2}}$ and $\gamma_{0}=1-\frac{u_{0}^{2}}{2 c^{2}}$.

In the dimensional form Eqn. (11) becomes:

$$
\frac{1}{\left(\omega-k u_{0}\right)^{2} \gamma_{3}-k^{2} V_{F e}^{2}-\frac{H^{2} k^{4} V_{F e}^{4} \gamma_{0}}{4 \omega_{p e}^{2}}}+\frac{\mu}{\left(\omega-k u_{0}\right)^{2} \gamma_{3}-\sigma k^{2} V_{F e}^{2}-\frac{\mu^{2} H^{2} k^{4} V_{F e}^{4} \gamma_{0}}{4 \omega_{p e}^{2}}}=\frac{1}{\omega_{p e}^{2}}
$$

Equation (11) is a quadratic equation in $\omega^{2}$ and has the solutions:

$$
\begin{aligned}
& \omega_{\text {fast }}=\left[\frac{1}{2 \gamma_{3}}\left((1+\mu)+(1+\sigma) k^{2}+\frac{\left(1+\mu^{2}\right) H^{2} k^{4} \gamma_{0}}{4}+\sqrt{D}\right)\right]^{\frac{1}{2}}+k u_{0} \\
& \omega_{\text {slow }}=\left[\frac{1}{2 \gamma_{3}}\left((1+\mu)+(1+\sigma) k^{2}+\frac{\left(1+\mu^{2}\right) H^{2} k^{4} \gamma_{0}}{4}-\sqrt{D}\right)\right]^{\frac{1}{2}}+k u_{0}
\end{aligned}
$$

where $D=4 \mu+\left\{(1-\mu)+(1-\sigma) k^{2}+\frac{\left(1-\mu^{2}\right) H^{2} k^{4} \gamma_{0}}{4}\right\}^{2}$ is the discriminant.

The discriminant ' $D$ ' in Equations (13) and (14) is always positive. Thus the solution for $\omega$ has two positive branches indicating the existence of two distinct electrostatic wave modes. In order to extract more information regarding the behavior of these two wave modes we have studied them numerically. It is shown that the linear dispersion curve for the fast mode [represented by Eq. (13)] depends significantly on $H$ but is almost independent of $\sigma$. On the other the linear dispersion of the slow mode [represented by Eq. (14)] depends slightly on $\sigma$ and is almost independent of $H$. The dependence of linear dispersion relation on the streaming velocity for both slow and fast mode is shown in Fig. 1. The phase velocity of both modes increases with increase in streaming velocity. Figure 2 shows the dependence of slow mode on quantum diffraction parameter $(H)$ and ion-to-electron temperature ratio. It is found that quantum diffraction has no significant effect on linear dispersion properties, 
whereas ion temperature has small but finite positive effect of increasing the frequency for a given wavenumber.

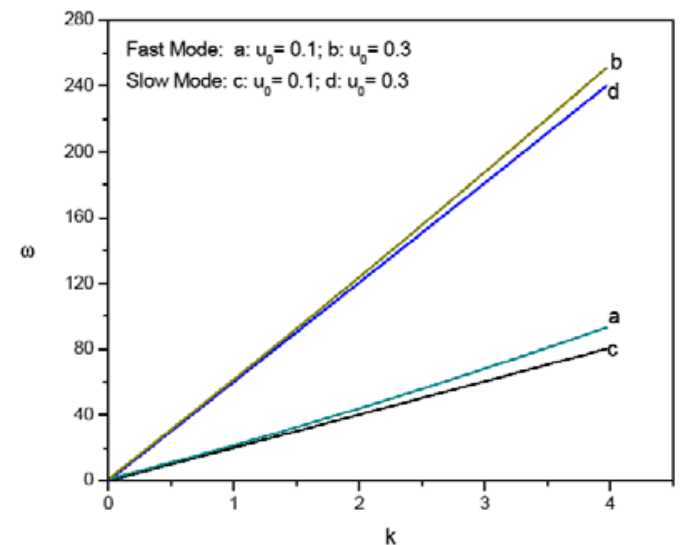

Figure 1. Dependence of Fast and Slow modes on relativistic streaming velocity $\left(u_{0}\right)$.

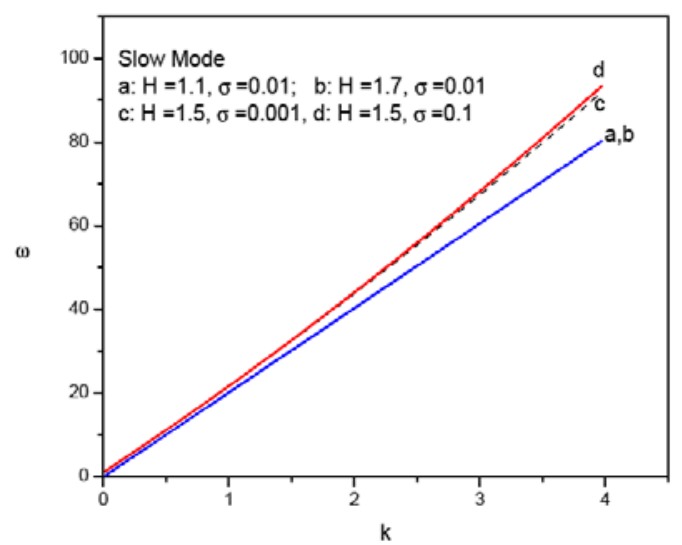

Figure 2. Dependence of Slow mode on quantum diffraction parameter $(H)$ and ion-to-electron temperature ratio $(\sigma)$.

If we assume that the ions are infinitely heavy compared to the electrons (i.e. $\mu=0$ ) then the wave branches (13) and (14) reduce respectively to:

and

$$
\omega_{\text {fast }}=\left[\frac{1}{2 \gamma_{3}}\left(1+k^{2}+\frac{H^{2} k^{4} \gamma_{0}}{4}\right)\right]^{\frac{1}{2}}+k u_{0}
$$

$$
\omega_{\text {slow }}=\left(\sqrt{\frac{\sigma}{\gamma_{3}}}+u_{0}\right) k
$$

In the dimensional form the frequencies of these two wave branches are given as:

$$
\omega_{\text {fast }}=\left[\frac{1}{2 \gamma_{3}}\left(\omega_{p e}^{2}+k^{2} V_{F e}^{2}+\frac{H^{2} k^{4} V_{F e}^{4} \gamma_{0}}{4 \omega_{p e}^{2}}\right)\right]^{\frac{1}{2}}+k u_{0}
$$

and

$$
\omega_{\text {slow }}=\left(\sqrt{\frac{\sigma}{\gamma_{3}}}+u_{0}\right) k
$$

Equation (17) corresponds to the dispersion relation for electron plasma waves including quantum diffractions and relativistic effects. The wave mode represented by Eq. (18) arises out of quantum statistical and relativistic effects. The fast mode corresponds to the usual electron plasma wave modified by quantum and relativistic effects. In the absence of the quantum diffraction (i.e. $H=0$ ) and relativistic effect dispersion relation (17) reduces to the well-known dispersion relation for electron plasma waves in classical plasma. The slow mode represented by (18) is of pure quantum origin and is caused by quantum statistical effect as is evident from the presence of the parameter $\sigma$. If both quantum statistical effect and relativistic effect are removed, the second mode vanishes.

\subsection{The KdV Equation}

In order to derive the desired $\mathrm{KdV}$ equation describing the nonlinear behavior of the plasma wave mode of pure quantum origin we use the standard reductive perturbation technique. We introduce the usual stretching of the space and time variables:

$$
\xi=\varepsilon^{1 / 2}(x-V t) \quad \text { and } \quad \tau=\varepsilon^{3 / 2} t
$$


where $V$ is the linear long wave phase speed normalized by electron Fermi speed $V_{F e}$ and $\varepsilon$ is a smallness parameter measuring the dispersion and nonlinear effects.

Equations (5)-(9) are written in terms of the stretched coordinates $\xi$ and $\tau$ and then the perturbation expansions (10) are substituted. Solving the lowest order equations with the boundary conditions that $n_{e}^{(1)}, u_{e}^{(1)}, n_{i}^{(1)}, u_{i}^{(1)}$ and $\phi^{(1)} \rightarrow 0$ as $|\xi| \rightarrow \infty$, the following solutions are obtained:

$$
n_{e}^{(1)}=\beta_{e} \phi^{(1)}, u_{e}^{(1)}=\alpha_{e} \phi^{(1)}, n_{i}^{(1)}=\beta_{i} \phi^{(1)} \text { and } u_{i}^{(1)}=\alpha_{e} \phi^{(1)}
$$

where

$$
\begin{aligned}
& \beta_{e}=-\frac{1}{\gamma_{3}\left(V-u_{0}\right)^{2}}\left[1-\frac{1}{\gamma_{3}\left(V-u_{0}\right)^{2}}\right]^{-1}, \beta_{i}=\frac{\mu}{\left(V-u_{0}\right)^{2}}\left[1-\frac{\sigma}{\left(V-u_{0}\right)^{2}}\right]^{-1} \\
& \alpha_{e}=-\frac{1}{\gamma_{3}\left(V-u_{0}\right)}\left[1-\left(\frac{1}{\left(V-u_{0}\right) \gamma_{3}}\right)^{2}\right]^{-1}, \alpha_{i}=\frac{\mu}{\left(V-u_{0}\right)}\left[1-\frac{\sigma}{\left(V-u_{0}\right)}\right]^{-1}
\end{aligned}
$$

Going for the next higher order terms in $\varepsilon$, after a few algebraic steps, the desired KdV equation is obtained:

$$
\frac{\partial \phi}{\partial \tau}+A \phi \frac{\partial \phi}{\partial \xi}+B \frac{\partial^{3} \phi}{\partial \xi^{3}}=0
$$

where

$$
A=\frac{F_{2}}{F_{1}} \text { and } B=\frac{F_{3}}{F_{1}}
$$

in which

$$
\begin{aligned}
& F_{1}=\left[\left(\gamma_{3} \alpha_{e}+\frac{\alpha_{i}}{\mu}\right)+\left(V-u_{0}\right)\left(\gamma_{3} \beta_{e}+\frac{\beta_{i}}{\mu}\right)\right] \\
& F_{2}=\left[\left(\gamma_{3} \alpha_{e}^{2}+\frac{\alpha_{i}^{2}}{\mu}\right)+\left(\beta_{e}^{2}+\frac{\sigma}{\mu} \beta_{i}^{2}\right)+2\left(V-u_{0}\right)\left(\gamma_{3} \alpha_{e} \beta_{e}+\frac{\alpha_{i} \beta_{i}}{\mu}-\alpha_{e}^{2} \gamma_{1}\right)\right] \\
& F_{3}=\left[\left(\frac{1-\sigma \gamma_{3}}{1+\mu \gamma_{3}}\right)-\left(\gamma_{0} \beta_{e}+\mu \beta_{i}\right) \frac{H^{2}}{4}\right]
\end{aligned}
$$

$\alpha_{e}, \beta_{e}, \alpha_{i}$ and $\beta_{i}$ are as defined earlier in equation (21) and $\gamma_{1}=\frac{u_{0}}{c^{2}}$.

It is to be noted that the coefficients of the dispersive and nonlinear terms in the KdV equation (22) get modified by the quantum and relativistic effects.

To find the steady state solution of Eq. (22) we transform the independent variables $\xi$ and $\tau$ into one variable $\eta=\xi-M \tau$ where $M$ is the normalized constant speed of the wave frame. Applying the boundary conditions that as $\eta \rightarrow \pm \infty, \phi, \frac{\partial \phi}{\partial \eta}, \frac{\partial^{2} \phi}{\partial^{2} \eta} \rightarrow 0$, the possible stationary solution of Eq. (22) is obtained as:

$$
\phi=\phi_{m} \sec h^{2}(\eta / \Delta)
$$

where the amplitude $\phi_{m}$ and width $\Delta$ of the soliton are given by:

$$
\phi_{m}=3 M / A
$$

and

$$
\Delta=\sqrt{4 B / M}
$$

The solitary wave structure is formed due to a balance between dispersive and nonlinear effects. Relative strength of these two effects determines the characteristic of such solitary wave structure. The coefficients $A$ and $B$, corresponding to the nonlinear effect and dispersive effect respectively, play 
crucial roles in determining the solitary wave structure. So it is important to study the dependence of these coefficients on different physical parameters. Numerical calculations show that the coefficient $A$ increases with increases in $\sigma$. It is almost independent of quantum diffraction parameter $H$ and streaming velocity $u_{0}$. The co-efficient $B$ of the dispersion term decreases significantly with increase in $\sigma$ but increases slightly with increase in the streaming velocity $u_{0}$. The coefficient $B$ depends interestingly on $H$. It decreases as $H$ is increased from zero and for certain critical value of $H$ (say $H_{c}$ ) it becomes zero. From equations (24) and (25) we get the critical value of $H$ as:

$$
H_{c}=2\left(\frac{1-\sigma \gamma_{3}}{1+\mu \gamma_{3}}\right)\left[\frac{\gamma_{0}}{\gamma_{3}\left(V-u_{0}\right)^{2}-1}+\frac{\mu^{2}}{\left(V-u_{0}\right)^{2}-\sigma}\right]^{-\frac{1}{2}}
$$

where $\gamma_{0}$ and $\gamma_{3}$ are as defined earlier. Numerically we find that the value of $H_{c}$ increases with increase in $u_{0}$ and decrease in $\sigma$. This result is very similar to that previously reported for ion acoustic solitary excitations in quantum plasma [22].

In this section using the quantum hydrodynamic model and reductive perturbation technique we have derived the Korteweg de Vries equation including the relativistic variation of mass of electrons with velocity for investigating small amplitude solitons. The dependence of the solitary wave structure on different plasma parameters such as relativistic streaming factor, ion-to-electron temperature ratio are studied both analytically and numerically. It is found that only compressive solitons can exist depending upon the values of different plasma parameters. For $H>H_{c}$ compressive solitons are obtained (Fig. $3)$.

Regarding the dependence of the amplitude and width of the soliton on the streaming velocity it is found that the soliton amplitude is almost independent of steaming velocity $u_{0}$ but with increase in streaming velocity the width increases. The soliton amplitude is independent of quantum diffraction parameter $H$. but with increasing $H$ the width shrinks for compressive solitons [Fig 4].

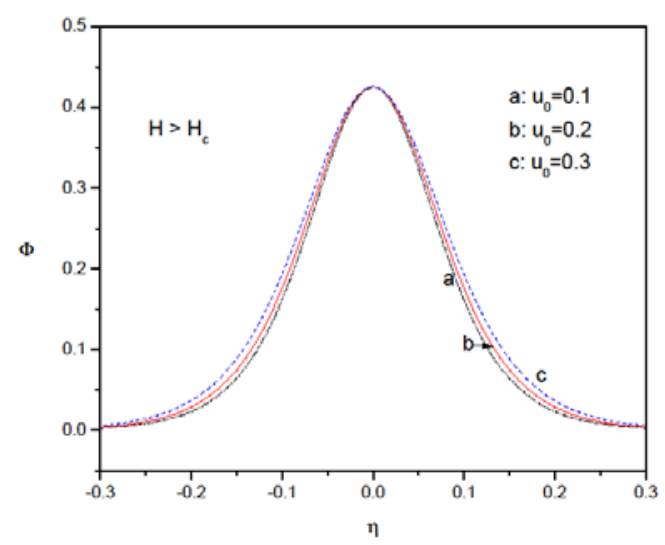

Figure 3. Compressive solitons for different values of relativistic streaming velocity $\left(u_{0}\right) ; H=2\left(>H_{c}\right)$, $\sigma=0.01$ and $M=0.1$.

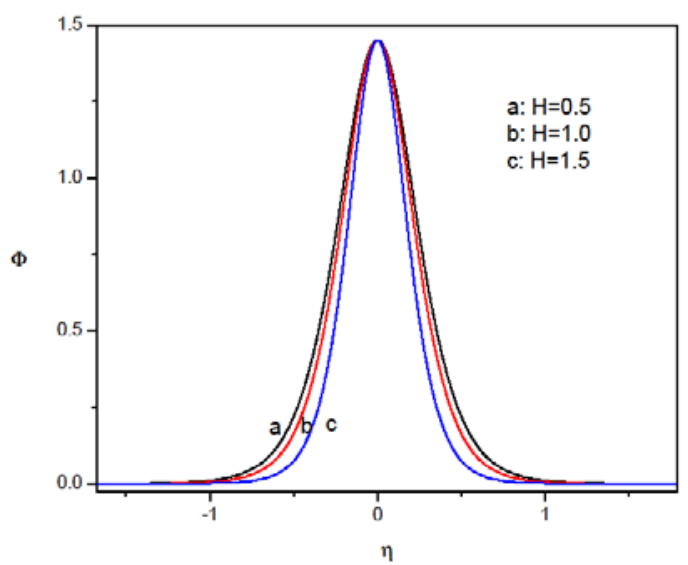

Figure 4. Compressive solitons for different quantum diffraction parameter $(H) ; u_{0}=0.2, \sigma=0.01$ and $M=0.1$. 


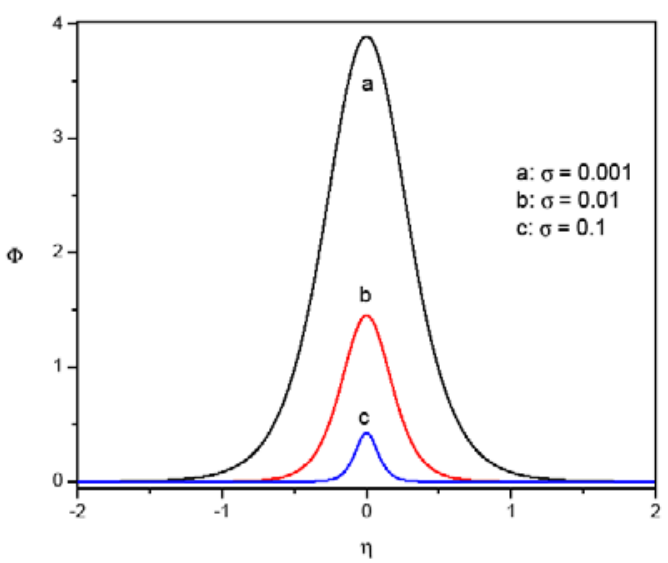

Figure 5. Compressive solitons for different ion to electron temperature ratio $(\sigma) ; H=1.5, u_{0}=0.2$ and $M=0.1$.

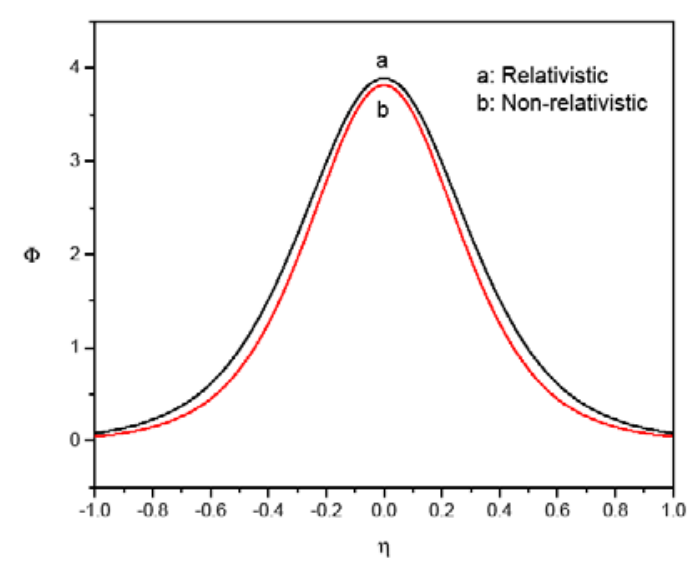

Figure 6. Effect of relativistic streaming velocity $\left(u_{0}\right)$ on the amplitude and width of compressive soliton $H=1.5, u_{0}=0.2, \sigma=0.01$ and $M=0.1$.

The dependence of soliton characteristics on ion to electron temperature ratio $\sigma$ is found to be significant. Both the amplitude and width of the solitons decrease significantly with increase in the value of $\sigma$ [Fig. 5]. Dependence of the solitary structure on the relativistic streaming factor is shown in Fig. 6 from which it is clear that relativistic effect slightly increases both the amplitude and width of the soliton.

\section{Effects of Relativistic Degeneracy: Chandrasekhar Treatment}

According to S. Chandrasekhar (1939) the electron degeneracy pressure in fully degenerate and relativistic configuration can be expressed in the following form:

$$
P_{j}=\left(\pi m_{e}^{4} c^{5} / 3 h^{3}\right)\left[R_{j}\left(2 R_{j}^{2}-3\right) \sqrt{1+R_{j}^{2}}+3 \sinh ^{-1} R_{j}\right]
$$

in which

$$
R_{j}=p_{F j} / m_{e} c=\left[3 h^{3} n_{j} / 8 \pi m_{e}^{3} c^{3}\right]^{\frac{1}{3}}=R_{j 0} n_{j}^{\frac{1}{3}}
$$

where $R_{j 0}=\left(n_{j 0} / n_{0}\right)^{1 / 3}$ with $n_{0}=8 \pi m_{e}^{3} c^{3} / 3 h^{3} \approx 5.9 \times 10^{29} \mathrm{~cm}^{-3}$, ' $c$ ' being the speed of light in vacuum. $p_{F j}$ is the electron Fermi relativistic momentum. It is to be noted that in the limits of very small and very large values of relativity parameter $R_{j}$, we obtain:

$$
\begin{gathered}
P_{j}=\frac{1}{20}\left(\frac{3}{\pi}\right)^{\frac{2}{3}} \frac{h^{2}}{m_{e}} n_{j}^{\frac{5}{3}} \quad\left(\text { for } R_{j} \rightarrow 0\right) \\
P_{j}=\frac{1}{8}\left(\frac{3}{\pi}\right)^{\frac{1}{3}} h c n_{j}^{\frac{4}{3}} \quad\left(\text { for } R_{j} \rightarrow \infty\right)
\end{gathered}
$$

Note that the degenerate electron pressure depends only on the electron number density but not on the electron temperature. Now considering the fact that $\frac{1}{n_{j}} \frac{\partial P_{j}}{\partial x}=\frac{\partial \sqrt{1+R_{j}^{2}}}{\partial x}$ the basic equations (2)- (4) can be written in the following normalized form:

$$
\frac{\partial\left(n_{j}\right)}{\partial t}+\frac{\partial\left(n_{j} u_{j}\right)}{\partial x}=0
$$




$$
\begin{gathered}
\left(\frac{\partial}{\partial t}+u_{j} \frac{\partial}{\partial x}\right) u_{j}=\frac{\partial \phi}{\partial x}-F_{j} \frac{\partial n_{j}}{\partial x}+\frac{H^{2}}{2} \frac{\partial}{\partial x}\left[\frac{1}{\sqrt{n_{j}}} \frac{\partial^{2} \sqrt{n_{j}}}{\partial x^{2}}\right] \\
\frac{\partial^{2} \phi}{\partial x^{2}}=\left(n_{e}-n_{i}\right)
\end{gathered}
$$

where $F_{j}=(\chi / 3)\left(R_{j 0}^{2} / \sqrt{1+R_{j 0}^{2}}\right)$ is the term arising from relativistic pressure in weakly relativistic case, whereas for ultra relativistic case $F_{j}=\chi R_{j 0} / 3$ where $\chi=m_{e} c^{2} / 2 k_{B} T_{F e}$. Here $j=e$ for electrons and $i$ for ions. $H$ is the non-dimensional quantum diffraction parameter defined as $H=\hbar \omega_{e} / 2 k_{B} T_{F e}$, where $T_{F e}$ is the Fermi temperatures for electrons; $n_{e 0}$ and $n_{i 0}$ are the equilibrium number densities electrons and ions respectively. The normalization has been carried out in the following manner:

$$
x \rightarrow x \omega_{e} / c_{s e}, \quad t \rightarrow t \omega_{e}, \quad \phi \rightarrow e \phi / 2 k_{B} T_{F e}, \quad n_{j} \rightarrow n_{j} / n_{j 0}, u_{j} \rightarrow u_{j} / c_{s}
$$

in which $\omega_{e}=\sqrt{4 \pi n_{e 0} e^{2} / m_{e}}$ is the cold electron plasma frequency, $c_{s h}=\sqrt{2 k_{B} T_{F e} / m_{e}}$ is the electronacoustic speed. It is to be noted that the parameter $R_{j 0}$ is a measure of the relativistic effects and may be called relativistic degeneracy parameter. For ultra relativistic case $R_{j 0} \gg 1$ and for weakly relativistic case $R_{j 0} \ll 1$. The parameter $R_{j 0}$ can also be related to mass density as $\rho\left(\mathrm{gr} / \mathrm{cm}^{3}\right)=1.97 \times 10^{6} \cdot R_{j 0}^{3}$ [Akbari-Moghanjoughi (2011)]. The density of white dwarfs can be in the range $10^{5}<\rho<10^{9}$. So in this case, the relativity parameter $R_{j 0}$ can be in the range $0.37<R_{j 0}<8$.

\subsection{Linear Dispersion Characteristics}

The dispersion relation for normalized wave frequency $\omega$ and wave number $k$, the following linear dispersion relation:

$$
\frac{1}{\omega^{2}-F_{e} k^{2}-\frac{H^{2} k^{4}}{4}}+\frac{\mu}{\omega^{2}-F_{i} k^{2}-\frac{\mu^{2} H^{2} k^{4}}{4}}=1
$$

where $F_{j}=\left(\chi R_{j 0}^{2} / \sqrt{1+R_{j 0}^{2}}\right) / 3$ in the weakly relativistic limit and $F_{j}=\chi R_{j 0} / 3$ in the ultra-relativistic case.

where

$$
\begin{aligned}
& \omega_{1}^{2}=\left[B+\sqrt{B^{2}-4 C}\right] / 2 \\
& \omega_{2}^{2}=\left[B-\sqrt{B^{2}-4 C}\right] / 2
\end{aligned}
$$

$$
\begin{aligned}
& B=k^{2}\left(F_{e}+F_{i}\right)+\left(H^{2} k^{4} / 2\right)+\left(1+\mu^{2}\right) \\
& C=\left(k^{2} F_{e}+H^{2} k^{4} / 4\right)\left(k^{2} F_{i}+\mu^{2} H^{2} k^{4} / 4\right)+k^{2}\left(F_{e}+F_{i}\right)+\left(H^{2} k^{4} / 4\right)\left(1+\mu^{2}\right)
\end{aligned}
$$

It indicates that two stable linear modes for EAWs are possible when one considers inertial and relativistic effects of both electrons and ions. EAWs are high frequency electrostatic electron oscillations where the restoring force comes from the electron pressure and the ions provide the inertia. If we neglect the inertia of electrons and assume that the pressure is solely due to the ultra-relativistic electrons then the dispersion relation (12) reduces to:

$$
\omega^{2}=\frac{k^{2}\left(\chi R_{e 0} / 3+H^{2} k^{2} / 4\right)}{1+k^{2}\left(\chi R_{e 0} / 3+H^{2} k^{2} / 4\right)}+\frac{H^{2} k^{4}}{4}
$$

In the long wavelength limit (i.e. $k \rightarrow 0$ )

$$
\omega=k \sqrt{\chi R_{e 0} / 3}
$$

The long wave phase speed is: 


$$
V_{0}=\omega / k=\sqrt{\chi R_{e 0} / 3}
$$

It represents the long wave dispersion character of EAWs in quantum-relativistic plasma composed of inertia less electrons and inertial cold ions. We numerically examine the behavior of the dispersion relation (37) with respect to the variations of $R_{e 0}$ and $H$. Fig. 7 shows the variation of $\omega$ with $k$ for different values of the relativity parameter $R_{e 0}$. It shows that the wave frequency $\omega$ increases with increase in the value of $R_{e 0}$. Fig. 8 shows the $\omega-k$ curves for different values of $H$. Obviously, the wave frequency $\omega$ also increases with increase in $H$.

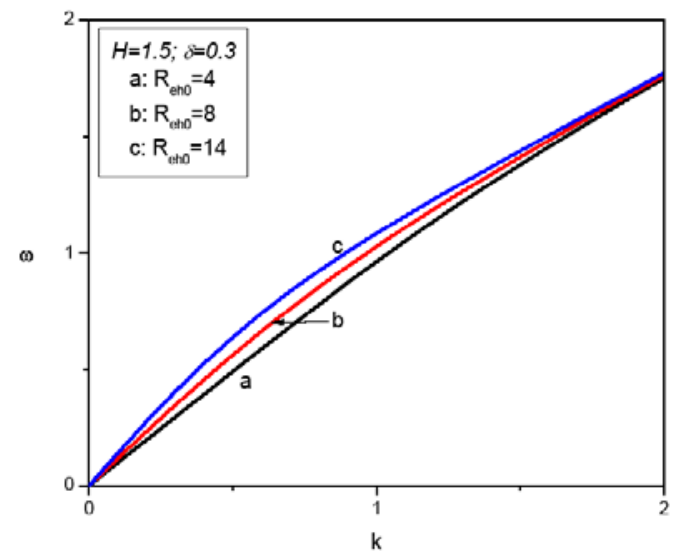

Figure 7. Dispersion Curve for different values of the relativity parameter $R_{e 0}$

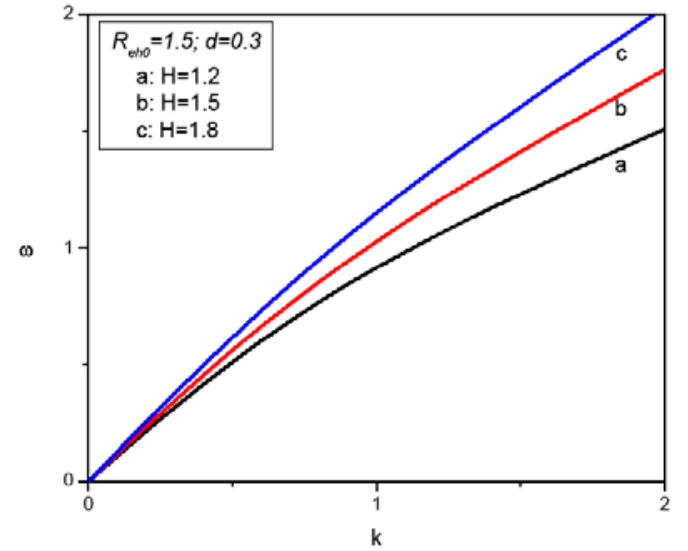

Figure 8. Dispersion Curve for different values of the quantum parameter $H$

\subsection{KdV Equation}

In order to study the nonlinear behavior of electron acoustic waves we consider inertia less hot ultra relativistic electrons, inertial cold electrons and stationary ions. The pressure effect is assumed to be only due to the hot electrons. This type of consideration has been made by many previous authors [3336]. Following the standard reductive perturbation technique we use the usual stretching of the space and time variables:

$$
\xi=\varepsilon^{1 / 2}\left(x-V_{0} t\right) \quad \text { and } \quad \tau=\varepsilon^{3 / 2} t
$$

where $V_{0}$ is the normalized linear long wave phase velocity given by Eq. (42) and $\varepsilon$ is the smallness parameter measuring the dispersion and nonlinear effects. Now writing the Equations (34)-(36) in terms of these stretched co-ordinates $\xi$ and $\tau$ and then applying the perturbation expansion (43) and solving for the lowest order equation with the boundary condition that $n_{e}^{(1)}, u_{e}^{(1)}, n_{e}^{(1)}, u_{e}^{(1)}$ and $\phi^{(1)} \rightarrow$ 0 as $|\xi| \rightarrow \infty$, the following solutions are obtained: 


$$
n_{e}^{(1)}=\frac{\delta \phi^{(1)}}{V_{0}^{2}}, \quad u_{e}^{(1)}=\frac{\delta \phi^{(1)}}{V_{0}}, \quad n_{i}^{(1)}=-\frac{\phi^{(1)}}{V_{0}^{2}}, \quad u_{i}^{(1)}=-\frac{\phi^{(1)}}{V_{0}}
$$

Going for the next higher order terms in $\varepsilon$ and following the usual method we obtain the desired Korteweg de Vries (KdV) equation:

$$
\frac{\partial \phi}{\partial \tau}+A \phi \frac{\partial \phi}{\partial \xi}+B \frac{\partial^{3} \phi}{\partial \xi^{3}}=0
$$

where

$$
A=-\frac{3}{2 V_{0}}=-\frac{3}{2 \sqrt{\delta \chi R_{e 0} / 3}} \text { and } B=\frac{V_{0}^{4}-2 H^{2} / 4}{2 V_{0}}=\frac{\left(\chi R_{e 0} / 3\right)^{2}-2 H^{2} / 4}{2 \sqrt{\chi R_{e 0} / 3}}
$$

Using usual techniques and applying the boundary conditions that as $\eta \rightarrow \pm \infty, \phi, \frac{\partial \phi}{\partial \eta}, \frac{\partial^{2} \phi}{\partial^{2} \eta} \rightarrow 0$, the possible stationary solution of Eq. (45) is obtained as:

$$
\phi=\phi_{m} \sec h^{2}(\eta / \Delta)
$$

where the amplitude $p h i_{m}$ and width $\Delta$ of the soliton are given by:

$$
\phi_{m}=3 M / A
$$

and

$$
\Delta=\sqrt{4 B / M}
$$

The solitary wave structure is formed due to a delicate balance between dispersive and nonlinear effects. Relative strength of these two effects determines the characteristic of such solitary wave structure. The coefficients $A$ and $B$, corresponding to the nonlinear effect and dispersive effect play crucial roles in determining the solitary wave structure. There exists a critical value of $R_{e 0}$ for which the solitary structure vanishes given by:

$$
\left(R_{e 0}\right)_{c}=3 H_{c} / \sqrt{2} \chi
$$

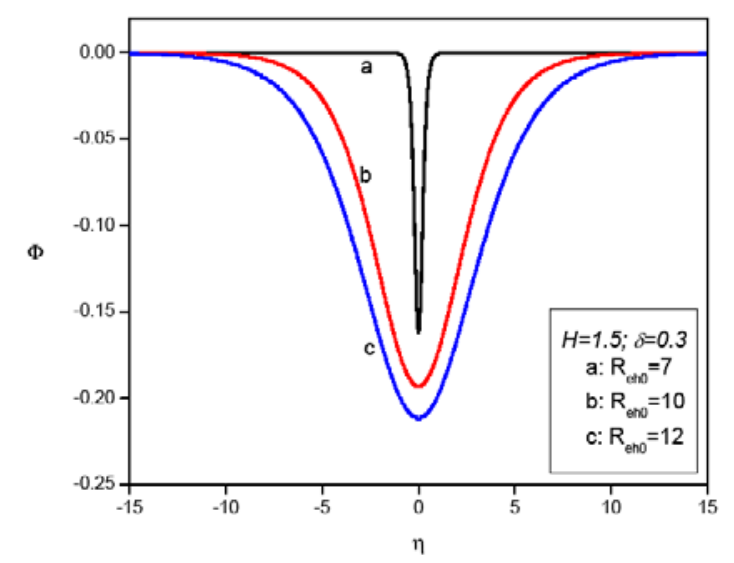

Figure 9. Solitary Structures for different values of the relativity parameter $R_{e 0}$ 


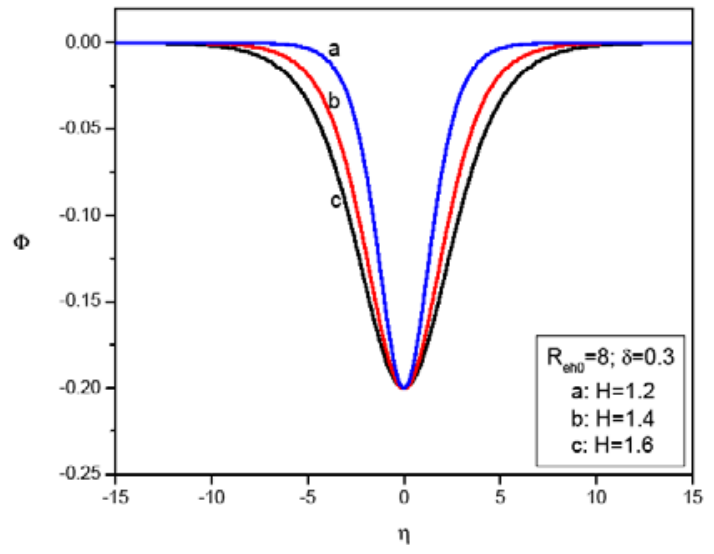

Figure 10. Solitary Structures for different values of the quantum parameter.

In this section we have investigated using the one-dimensional quantum hydrodynamic model and the standard reductive perturbation technique both the linear and nonlinear properties of electron-acoustic waves in two-component relativistically degenerate plasma consisting of electrons and ions. A general type dispersion relation has been obtained including inertia and quantum relativistic effects of both electrons and ions. It is shown that two stable linear modes of propagation are possible for electronacoustic waves when one considers the inertia effect of both species. The wave frequency is shown to increase with the increase in the values of relativity parameter $R_{e 0}$ and also the quantum diffraction parameter $H$.

To study the nonlinear behavior of the wave a $\mathrm{KdV}$ equation has been derived in which the coefficients of the nonlinear and dispersive terms are found to get modified due to the inclusion of quantum relativistic effects. There exists a critical value of the relativistic degeneracy parameter $R_{\text {eh } 0}$ such that for $R_{e 0}<\left(R_{e 0}\right)_{c}$ no soliton solution is possible. This critical value of the degeneracy parameter is determined by the values of $H$. From Equations (46)- (49) it is obvious that the degenerate plasma under consideration supports only rarefactive solitary wave structures which are associated with negative potentials. Figure 9 shows electron-acoustic solitary profiles for different values of the relativistic degeneracy parameter $R_{e 0}$ (which is directly proportional to the plasma number density) for fixed values of $M$ and $H$. It shows that both the amplitude and width of the soliton increase with increase of $R_{e 0}$. Fig. 10 shows solitary structures for different values of $H$ keeping other parameters fixed. It shows that the soliton width increases with increase in the value of $H$ but its amplitude is independent of $H$. The amplitude of electron-acoustic solitary structure increases with increase in $R_{e 0}$ but it is independent of $H$. On the other hand the width of the soliton increases with increase in $R_{e 0}$ or $H$.

\section{Relativistic Drifts and Relativistic Degeneracy: A Comparison}

A comparison of these two kinds of relativistic effects is discussed. When the relativistic effects are due to streaming motion only compressive solitons are observed. The properties and dependence on streaming velocity $u_{0}$, quantum diffraction $H$ and ion temperature are discussed in section 5 . In section 6 the investigation of linear and nonlinear propagation characteristics of EAWs are carried out in relativistic degenerate dense plasma consisting of electrons and ions. It is shown that the plasma under consideration can support only rarefactive solitary waves under certain restricted regions of plasma parameters. The soliton properties are shown to depend significantly on the relativistic degeneracy parameter $R_{e 0}$ and also the quantum diffraction parameter $H$.

The present investigation may be helpful in understanding the basic features of electron-acoustic waves in super dense astrophysical objects like white dwarfs, neutron stars as well as in the future 
intense laser-solid plasma experiments where the relativistic electron degeneracy effects become important.

Acknowledgement. The author would like to thank CSIR \& DST, Govt. of India for providing financial assistance and JIS University, Kolkata and Jadavpur University, Kolkata, India to carry out this research work.

\section{References}

1. G. Manfredi, "How to model quantum plasmas" Fields Institute Communications, vol. 46, 263, 2005.

2. M. Opher, L. O. Silva, D. E. Dauger, V. K. Decyk and J. M. Dawson, "Nuclear reaction rates and energy in stellar plasmas: The effect of highly damped modes" Physics of Plasmas, vol. 8, 2454, 2001.

3. A. Markowich, C. Ringhofer and C. Schmeiser, Semiconductor Equations, Springer, Vienna, 1990.

4. K. F. Berggren and Z.-L.Ji, "Quantum chaos in nano-sized billiards in layered two-dimensional semiconductor structures", Chaos, vol. 6, 543, 1996.

5. W. L. Barnes, A. Dereux and T. W. Ebbesen, "Surface plasmon subwavelength optics" Nature (London), vol. 424, 824, 2003.

6. T. C. Killian, "Cool Vibes", Nature (London) vol.441, 297, 2006.

7. G. Chabrier, F. Douchin and A. Y. Potekhin, "Dense astrophysical plasmas" Journal of Physics: Condensed Matter, vol.14 9133, 2002.

8. K. H. Becker, K. H. Schoenbach and J. G. Eden, "Microplasmas and applications", Journal of Physics D: Applied Physics, vol. 39, R55, 2006.

9. L. K. Ang, W. S. Koh, Y. Y. Lau and T. J. T. Kwan, "Space-charge-limited flows in the quantum regime", Physics of Plasmas, vol. 13 056701, 2006.

10. L. K. Ang and P. Zhang, "Ultrashort-Pulse Child-Langmuir Law in the Quantum and Relativistic Regimes", Physical Review Letters, vol. 98, 164802, 2007.

11. C. Grabbe, "Wave propagation effects of broadband electrostatic noise in the magnetotail", Journal of Geophysical Research, vol. 94, 17299, 1989 .

12. J. I. Vette, Summary of Particle Population in the Magnetosphere, Reidel, Dordrecht, p. 305, 1989.

13. H. Ikezi, "Experiments on ion-acoustic solitary waves", Physics of Fluids, vol. 16, 1668, 1973.

14. B. Shen and J. Meyer-ter-Vehn, "Pair and $\curlyvee$-photon production from a thin foil confined by two laser pulses", Physical Review E, vol. 65, 016405, 2001.

15. E. P. Liang, S. C. Wilks, and M. Tabak, "Pair Production by Ultraintense Lasers", Physical Review Letters, vol. 81, 4887, 1998.

16. K. A. Holcomb, T.Tajima, "General-Relativistic Plasma Physics in the Early Universe", Physical Review D, vol. 40, 3809, 1989

17. R. Saeed, A. Shah, M. N. Ha, "Nonlinear Korteweg-de Vries equation for soliton propagation in relativistic electron-positron-ion plasma with thermal ions", Physics of Plasmas, vol. 17, 102301, 2010.

18. S. K. El-Labany, M.S. Abdel Krim, S.A. El-Warraki, W.F. El-Taibany, "Modulational instability of a weakly relativistic ion acoustic wave in a warm plasma with nonthermal electrons", Chinese Physics, vol.12, 759, 2003.

19. R. Bharuthram, M.Y.Yu, "Relativistic electron plasma waves", Astrophysics and Space Sciences, vol. 207, 197, 1993.

20. S. I. Shapiro, S.A.Teukolsky, Black Holes, White Dwarfs and Neutron Stars, John Wiley \& sons, New York, 1983.

21. M. Marklund, P.K.Shukla, "Kinetic theory of electromagnetic ion waves in relativistic plasmas"Review of Modern Physics, vol. 78, 591, 2006.

22. F. Haas, L. G. Garcia, J. Goedert, and G. Manfredi, "Quantum ion-acoustic waves" Physics of Plasmas, vol. 10, 3858, 2003 .

23. L.S. Stenflo, P.K. Shukla and M. Marklund, "New low-frequency oscillations in quantum dusty plasmas", Europhysics Letters, vol. 74, no. 5, 844, 2006.

24. C.L. Gardner and C. Ringhofer, "Smooth quantum potential for the hydrodynamic model" Physical Review E, vol. 53,157, 1996.

25. P. K. Shukla and B. Eliasson, "Formation and Dynamics of Dark Solitons and Vortices in Quantum Electron Plasmas", Physical Review Letters, vol. 96, 245001, 2006. 
26. S. A. Khan and A. Mushtaq, "Linear and nonlinear dust ion acoustic waves in ultracold quantum dusty plasmas", Physics of Plasmas, vol. 14, 083703, 2007.

27. B. Sahu and R. Roychoudhury, "Cylindrical and spherical quantum ion acoustic waves" Physics of Plasmas, vol.14, 012304, 2007.

28. B. Sahu and R. Roychoudhury, "Electron acoustic solitons in a relativistic plasma with nonthermal electrons", Physics of Plasmas, vol. 13, 072302, 2006.

29. S. Ali and P. K. Shukla, "Dust acoustic solitary waves in a quantum plasma", Physics of Plasmas, vol. 13, $022313,2006$.

30. P. K. Shukla and S. Ali, "Dust acoustic waves in quantum plasmas", Physics of Plasmas, vol.12, 114502, 2005.

31. B. Ghosh, S. Chandra \& S.N.Paul, "Amplitude modulation of electron plasma waves in a quantum plasma", Physics of Plasmas, vol. 18, 012106, 2011. 